\title{
Landesverweisung und Freizügigkeitsabkommen - Bundesgericht vertagt Entscheid über Normenkonflikt
}

Kommentar zu den Urteilen des Bundesgerichts 6B_1152/2017 vom 28. November 2018 und 6B_235/2018 vom 1. November 2018

Nina Burri *

Der vorliegende Beitrag bespricht die ersten zwei Urteile des Bundesgerichts zum Verhältnis der neuen Landesverweisung und dem Freizügigkeitsabkommen (FZA). Die Richtungsweisung des Bundesgerichts wurde aufgrund der grossen Relevanz des Themas in der Praxis mit Spannung erwartet. Doch das Warten hat sich (noch) nicht gelohnt. In beiden Urteilen kann sich das Bundesgericht einer eingehenden Prüfung der Vorrangsfrage entziehen. Den ersten Fall (obligatorische Landesverweisung) weist das Bundesgericht erwartungsgemäss ans Zürcher Obergericht zurück, da Letzteres verkannt hatte, dass sich der Beschwerdeführer nicht auf das FZA berufen kann. Im zweiten Fall hält das Bundesgericht fest, dass die Vorinstanz Art. 66abis StGB (fakultative Landesverweisung) FZA-konform angewandt habe. Die Begründung des zweiten Falles erstaunt allerdings aufgrund einer widersprüchlichen Vorgehensweise und einiger sachlich schwer nachvollziehbarer Aussagen, die am Ende des vorliegenden Beitrags kommentiert werden.

I. Vorspann .65

II. Auftritt des Bundesgerichts.

1. Akt: Urteil 6B_1152/2017 vom 28. November 2018

2. Akt: Urteil 6B_235/2018 vom 1. November 2018 (zur Publikation vorgesehen) 67

III. Ausblick

Zitiervorschlag: Nina Burri, Landeserweisung und Freizügigkeitsabkommen Bundesgericht vertagt Entscheid über Normenkonflikt, in: suigeneris 2019, S. 64

URL: $\quad$ sui-generis.ch/93

DOI: $\quad$ https://doi.org/10.21257/sg.93

* Nina Burri, Dr. iur., Rechtsanwältin (nina.burri@icc-cpi.int), ehemalige Gerichtsschreiberin am Bezirksgericht Zürich, zurzeit Associate Trial Lawyer (Assistenz-Staatsanwältin) im Büro der Chefanklägerin am Internationalen Strafgerichtshof in Den Haag. Nina Burri vertritt in diesem Beitrag ihre persönliche Meinung. Sie dankt Dr. des. Barbara Kammermann für die kritische Durchsicht des Entwurfs und die wertvollen Hinweise.

Dieses Werk ist lizenziert unter einer Creative Commons Namensnennung - Weitergabe unter gleichen Bedingungen 4.0 International Lizenz. 


\section{Vorspann}

1 Am 1. Oktober 2016 trat die Ausführungsgesetzgebung zur Ausschaffungsinitiative in Kraft. ${ }^{1}$ Seither spülten die Strafverfolgungsbehörden zahlreiche Fälle in die Mühlen der Gerichte, in denen die Landesverweisung verurteilter Straftäter² zu prüfen war.

Bei der Anwendung von Art. 66a ff. StGB kamen in der Praxis, wie bei neuen Gesetzesbestimmungen üblich, erstmalige Sach- und Rechtsfragen auf. Eine häufig auftretende Problematik ist die potentielle Landesverweisung von Straftätern, die Ansprüche aus dem FZA3 und dem EFTA-Übereinkommen 4 ableiten können.

Namentlich sieht das FZA andere Kriterien für eine Einschränkung von Aufenthalts- und Einreiserechten vor, als neu in Art. 66a ff. StGB festgehalten sind. Es stellt sich daher die Frage, welche Bestimmungen vorgehen bzw. ob und wie

weizerisches Strafgesetzbuch und Militärstrafgesetz (Umsetzung von Art. 121 Abs. 3-6 BV über die Ausschaffung krimineller Ausländerinnen und Ausländer), Änderung vom 20. März 2015, BBl 20152735 ff. Die Inkraftsetzung der Ausführungsbestimmungen folgte am 1. März 2017 (AS 20162329 und AS 2017 563).

2 Aus Gründen der Lesbarkeit wurde im Text die männliche Form gewählt, nichtsdestoweniger beziehen sich die Angaben auf Angehörige beider Geschlechter.

3 Abkommen vom 21. Juni 1999 zwischen der Europäischen Gemeinschaft und ihren Mitgliedstaaten einerseits und der Schweizerischen Eidgenossenschaft andererseits über die Freizügigkeit (FZA; SR 0.142.112.681).

4 Übereinkommen vom 4. Januar 1960 zur Errichtung der Europäischen Freihandelsassoziation (EFTA) in der konsolidierten Fassung des Vaduzer Abkommens vom 21. Juni 2001 (SR 0.632.31), Anhang K und Anhang K - Anlage 1. In diesem Beitrag wird aus Gründen der Leserfreundlichkeit jeweils nur auf die Bestimmungen des FZA und nicht auch auf die gleichlautenden Bestimmungen des EFTA-Übereinkommens verwiesen. diese zwei verschiedenen Einschränkungsmechanismen des Aufenthaltsrechts freizügigkeitsberechtigter Ausländer in der Schweiz rechtsdogmatisch miteinander in Einklang zu bringen sind. 5

\section{Auftritt des Bundesgerichts}

4 Rund zwei Jahre nach Inkrafttreten der neuen Gesetzgebung äusserte sich das Bundesgericht nun erstmals zu dieser Thematik. Es tat dies anlässlich zweier Fälle, die ein knappes Jahr zuvor das Zürcher Obergericht entzweit hatten. ${ }^{6}$

5 Diese erste höchstrichterliche Richtungsweisung war mit Spannung erwartet worden. Doch das Warten hat sich (noch) nicht gelohnt. Das Bundesgericht weist einen Fall zurück ans Zürcher Obergericht und entzieht sich im anderen Fall einer vertieften fachlichen Prüfung des Normenkonflikts. Der Entscheid über die Vorrangsfrage bezüglich Landesverweisung und FZA wurde somit vorerst vertagt.

6 Im Folgenden werden die zwei Urteile sowie die ihnen zugrundeliegenden vorinstanzlichen Entscheide kurz zusammengefasst. Anschliessend kommentiert die Autorin ausgewählte Erwägungen. Am Ende folgt ein kurzer Ausblick über die Auswirkungen der Urteile auf die künftige Rechtsanwendung.

5 Siehe hierzu ausführlich Nina Burri und Valerio Priuli, Landesverweisung und Freizügigkeitsabkommen, AJP 07/2017, S. 886 ff., 887-889 sowie Sabine Gless, Anna Petrig und Christa Tobler, Ein fachübergreifendes Prüfprogramm für die obligatorische Landesverweisung nach Art. 66a StGB, forumpoenale 2/2018, S. $97 \mathrm{ff}$.

6 Urteil des Zürcher Obergerichts (I. Strafkammer) SB170250 vom 22. August 2017; Urteil des Zürcher Obergerichts (II. Strafkammer) SB170315 vom 16. Januar 2018. Vgl. Thomas Hasler, Landesverweisung spaltet Obergericht, Tages-Anzeiger vom 17. Januar 2018. 
1. Akt: Urteil 6B_1152/2017 vom 28. November 2018

7 Der zuerst beim Bundesgericht eingegangene Fall betrifft einen deutschen Staatsangehörigen $\mathrm{C}$ ohne festen Wohnsitz in der Schweiz. C wurde am 4. Mai 2017 vom Bezirksgericht Winterthur wegen Angriffs (Art. 134 StGB) zu einer bedingten achtmonatigen Freiheitsstrafe unter Ansetzung einer Probezeit von vier Jahren verurteilt. Gleichzeitig verwies das Bezirksgericht Winterthur ihn für fünf Jahre des Landes.

8 Gegen den Entscheid wurde in der Folge Berufung beim Obergericht Zürich erhoben. Dieses setzte sich in seinem Urteil mit dem Normenkonflikt zwischen Art. 5 Anhang I FZA und Art. 66a ff. StGB auseinander und hielt unter Hinweis auf BGE 142 II 35 und die einschlägige Lehre fest, dass das FZA vorgehe. Auf den Einzelfall angewendet kam es zum Schluss, dass das vorliegende persönliche Verhalten des Beschuldigten nicht für eine Einschränkung der Freizügigkeitsrechte nach Art. 5 Anhang I FZA genüge. Es hob daher die obligatorische Landesverweisung auf.7 Die Oberstaatanwaltschaft Zürich zog das Urteil daraufhin ans Bundesgericht weiter.

9 Das Bundesgericht weist den Fall nun wieder zurück ans Zürcher Obergericht. Es begründet dies damit, dass der Beschuldigte zwar deutscher Staatsbürger sei, das Obergericht aber verkannt habe, dass der Beschuldigte gar keine Ansprüche aus dem FZA ableiten könne.
Urteil des Zürcher Obergerichts (I. Strafkammer) SB170250 vom 22. August 2017, E. 3.2. und 4.
10 Der Beschuldigte sei vor der Anlasstat seit sieben Jahren nicht mehr in der Schweiz angemeldet gewesen und verfüge weder über eine Arbeits- noch eine Aufenthaltsbewilligung. Auch mit der in der Schweiz wohnhaften Familie habe er lediglich sporadischen Kontakt. Ferner seien keine Tatsachen vorgebracht worden, weshalb dem Beschuldigten das Leben oder Arbeiten in der Schweiz wichtig gewesen wären. Es sei daher unter keinem Titel des FZA ein Aufenthaltsrecht des Beschwerdegegners ersichtlich. Damit sei auch die Vorrangfrage zwischen dem FZA und der Landesverweisung nach StGB nicht zu prüfen und der Fall zur Prüfung der Anwendbarkeit von Art. 66a ff. StGB ans Obergericht zurückzuweisen. ${ }^{8}$

\section{Kommentar}

11 Dieser Entscheid war absehbar. Denn die Prüfung des Obergerichts Zürich, ob sich der Beschuldigte auf einen Aufenthaltstitel aus dem FZA berufen könne, war in der Tat zu knapp ausgefallen. 9

12 Richtigerweise hielt das Bundesgericht im Einklang mit der Rechtsprechung seiner II. öffentlich-rechtlichen Kammer fest, dass das FZA lediglich zu einem doppelt bedingten Aufenthalt in der Schweiz berechtige. ${ }^{10}$ Nämlich einerseits nach Massgabe der spezifischen Vertragsvereinbarungen als Voraussetzung eines rechtmässigen Aufenthalts und an-

8 Urteil des Bundesgerichts 6B_1152/2017 vom 28. November 2018, E. 2.3.5. f.

9 Urteil des Zürcher Obergerichts (I. Strafkammer, Fn. 6), E. 3.1. Siehe auch Christa Tobler, Das Freizügigkeitsabkommen impliziert kein allgemeines Aufenthaltsrecht, NZZ vom 9. November 2017.

10 Urteil des Bundesgerichts 6B_1152/2017 vom 28. November 2018, E. 2.5.2. mit Hinweisen auf BGE 144 II 1, BGE 139 II 121 und Urteil des Bundesgerichts 2C_1005/2017 vom 20. August 2018. 
dererseits nach Massgabe des rechtskonformen Verhaltens im Sinne von Art. 5 Anhang I FZA. Da vorliegend die Voraussetzungen eines rechtmässigen Aufenthaltsanspruchs aus dem FZA wohl nicht erfüllt waren, musste Art. 5 Anhang I FZA nicht geprüft werden. ${ }^{11}$

13 Dieses Urteil ist folgerichtig und erinnert die unteren Instanzen daran, dass der Anspruch auf einen FZA-Aufenthaltstitel nicht alleine aus einer EU-Staatsangehörigkeit abgeleitet werden kann.

14 Offen bleibt aber, was passiert, wenn sich der Beschuldigte im Rechtsmittelverfahren oder danach aufgrund veränderter Umstände plötzlich auf das FZA berufen könnte (wenn er sich beispielsweise auf Stellensuche begibt oder sich selbständig machen würde). ${ }^{12}$

Die Anwendbarkeit des FZA ist eine Rechtsfrage und kann somit vor Rechtskraft einer Landesverweisung vor allen Instanzen vorgebracht werden. Das Vorliegen veränderter tatsächlicher Umstände (z.B. das Pflegen verwandtschaftlicher Beziehungen, finanzielle Mittel oder Bemühungen für eine Stellensuche) ist hingegen eine Sachfrage, die im Rechtsmittelverfahren nur beschränkt vorgebracht werden kann. Nach einer rechts-

$11 \overline{\text { Aufgrund der Angaben im Sachverhalt hätte das }}$ Obergericht insbesondere prüfen müssen, ob $\mathrm{C}$ ein Recht auf Einreise oder einen voraussetzungslosen dreimonatigen Aufenthalt aus dem FZA ableiten konnte (vgl. BGE 143 IV 97). Die bundesgerichtliche Feststellung, dass «unter keinem Titel» ein Aufenthaltsrecht des Beschwerdegegners ersichtlich sei, ist daher ebenso vorauseilend und überzeugt nicht vollends. Für eine Übersicht der $\mathrm{zu}$ prüfenden Kriterien siehe Gless/Petrig/Tobler (Fn. 5), S. 97 ff.

12 Vgl. Luzia Vetterli, Verhältnis Freizügigkeit und Landesverweisung: zwei Urteile, ius.focus. Januar 2019, Heft 1. kräftigen Verurteilung richtet sich das Vorgehen nach kantonalem Recht, wobei die Praxis noch zeigen muss, in welchem Verfahren die erneute bzw. nachträgliche Prüfung der Zulässigkeit einer Einschränkung von Art. 5 Anhang I FZA vorzunehmen ist. ${ }^{13}$

\section{Akt: Urteil 6B_235/2018 vom} 1. November 2018 (zur Publikation vorgesehen)

16 Die zweite Beschwerde richtete sich gegen ein Urteil des Zürcher Obergerichts vom 16. Januar 2018 und bot Anlass zu einer ausführlicheren Prüfung. Betroffen war der schwedisch-serbische Doppelbürger A mit Aufenthaltsbewilligung B. A hatte einer anderen Person im Laufe einer Auseinandersetzung gedroht sie umzubringen und ihr aus drei Metern Distanz eine leere «Smirnoff»-Flasche an den Kopf geworfen. Der Angegriffene erlitt dabei eine stark blutende Rissquetschwunde am Kopf.

17 Das Bezirksgericht Zürich verurteilte A wegen qualifizierter einfacher Körperverletzung und Drohung (Art.123 Ziff. 1 Abs. 1 und Ziff. 2 Abs. 2 StGB sowie Art. 180 StGB) zu acht Monaten Freiheitsstrafe, bedingt aufgeschoben unter Ansetzung einer Probezeit von vier Jahren. Ferner widerrief es zwei bedingt

$13 \overline{\text { Im Kanton Zürich etwa ist gemäss einer Weisung }}$ des Migrationsamts beim Strafgericht ein Revisionsgesuch einzureichen, da es den Vollzugsbehörden verwehrt ist, eine von einem Strafgericht angeordnete strafrechtliche Massnahme abzuändern oder in Wiedererwägung zu ziehen. Dieser Ansatz ist jedoch fraglich, bedingt ein Revisionsgesuch doch Tatsachen, die vor dem Urteil stattgefunden haben. Denkbar wäre daher eher ein Nachverfahren im Sinne von Art. 363 ff. StPO. Vgl. Weisung «Landesverweisung» des Zürcher Migrationsamts vom 4. Februar 2019, S. 12; Burri/Priuli (Fn. 5), S. 897. 
ausgefällte Geldstrafen aus den Jahren 2014 und 2015. Da es sich weder bei der einfachen Körperverletzung noch bei der Drohung um Katalogtaten im Sinne von Art. 66a StGB handelt, hatte das Bezirksgericht Zürich die obligatorische Landesverweisung nicht zu prüfen. Von der Anordnung einer fakultativen Landesverweisung im Sinne von Art. 66a ${ }^{\text {bis }}$ StGB sah es ab.

18 Die Oberstaatsanwaltschaft erhob dagegen Berufung und beantragte den Vollzug der Freiheitsstrafe sowie einen fakultativen Landesverweis von fünf Jahren. Die II. Strafkammer des Obergerichts Zürich folgte im Januar 2018 dem Antrag der Oberstaatsanwaltschaft, verwies A jedoch nur für drei Jahre des Landes. ${ }^{14}$

In einer sehr ausführlichen Begründung befasste sich die Kammer sowohl mit der Vorrangsfrage als auch der Auslegung von Art. 5 Anhang I FZA. ${ }^{15}$ Sie hielt dabei - anders als noch die I. Strafkammer sechs Monate zuvor - fest, dass die Ausnahmeregel nach BGE 142 II 35 nicht überzeuge und die Vorrangsfrage daher nach Schubertpraxis zu prüfen sei. ${ }^{16}$ Dabei komme den StGB-Bestimmungen über die neue Landesverweisung Vorrang $\mathrm{zu}$.

In Übereinstimmung mit der bisherigen Bundesgerichtsrechtsprechung hielt die

14 Urteil des Zürcher Obergerichts (II. Strafkammer) SB170315 vom 16. Januar 2018.

15 Auffallend ist dabei, dass die Prüfung der Frage, ob sich der Beschuldigte auf das FZA berufen kann, ähnlich wie beim vorhergehend besprochenen Fall wiederum sehr knapp ausfiel. Ebenda, E. IV.2.a).

16 Die II. Strafkammer ging hier fälschlicherweise auch betreffend Art. 66a ${ }^{\text {bis }}$ StGB von einem direkten Normenkonflikt aus. Ebenda, E. IV.2.c) bb). Siehe hierzu unten, S. 70, Fn. 27.
II. Strafkammer aber fest, dass von der Auslegung des EuGH zu im FZA enthaltenen, aus dem EU-Recht übernommenen Bestimmungen nur aus triftigen Gründen abzuweichen sei. ${ }^{17}$ Gleichzeitig rief sie zu einer gewissen Zurückhaltung der Anwendung der obligatorischen Landesverweisung gegenüber FZAAnspruchsberechtigten ${ }^{18}$ auf, und erklärte, dass die fakultative Landesverweisung nur zulässig sei, wenn von der verwiesenen Person eine Gefahr für die öffentliche Sicherheit und Ordnung ausgehe. Der Gefährdungsbegriff sei hierbei eigenständig auszulegen. ${ }^{19}$

21 Schliesslich nahm die Kammer eine der Härtefallklausel ähnliche Verhältnismässigkeitsabwägung zwischen den Auswirkungen der Landesverweisung auf das Leben des Beschuldigten und den öffentlichen Interessen vor. Im Rahmen der Abwägung der öffentlichen Interessen verwies sie unter anderem auf die Begründung des unbedingten Strafvollzugs, die im Wesentlichen auf die erhöhte Rückfallgefahr des Beschuldigten abstellte. ${ }^{20}$ Die II. Strafkammer nahm somit unter dem Titel der Verhältnismässigkeit der fakultativen Landesverweisung nach StGB implizit auch die in Art. 5 Anhang I FZA vorgesehene Gefährdungsprüfung vor.

Vor Bundesgericht wurde die gegen diesen Entscheid erhobene Beschwerde in Fünferbesetzung behandelt. D.h., die Bundesrichter - oder zumindest ein Bundesrichter

17 Ebenda, E. IV.2.b) ff. mit weiteren Hinweisen.

18 Das Urteil erwähnt wiederholt den Begriff der «Bürger von EU-Staaten». Korrekterweise sollte hier auf FZA-berechtigte Personen verwiesen werden, können dies doch auch Drittstaatsangehörige sein. Ebenda, E. IV.2.c). Siehe hierzu unten, S. 72.

19 Ebenda, E. IV.3.c).

20 Ebenda, E. III.3. 
oder eine Bundesrichterin - erachteten die in der Beschwerde aufgeworfenen Rechtsfragen als solche von grundsätzlicher Bedeutung (Art. 20 BGG).

Vorweg hält das Bundesgericht fest, dass einer Beschwerde in Strafsachen gegen eine Landesverweisung (Art.66a StGB) in analoger Anwendung von Art. 103 Abs. 2 lit.b BGG von Gesetzes wegen aufschiebende Wirkung zukomme. ${ }^{21}$ Es erübrigt sich in der Praxis folglich ein gesondertes Gesuch um aufschiebende Wirkung.

Schliesslich öffnet das Bundesgericht den Vorhang für die lange erwarteten Erwägungen: Es führt aus, dass sich $\mathrm{A}$ als schwedischer Staatsangehöriger grundsätzlich auf das FZA berufen könne. Dann erläutert es knapp die Ziele des FZA und die Einschränkungen gemäss Art. 5 Anhang I FZA. Das Bundesgericht verweist hier wiederum auf den doppelt bedingten Aufenthaltsanspruch und führt aus, dass sich der schuldig gesprochene Straftäter evidentermassen nicht an diese Konformitätsbedingungen gehalten habe. Das FZA enthalte keine strafrechtlichen Bestimmungen und sei kein strafrechtliches Abkommen. Die Schweiz sei in der Legiferierung des Strafrechts auf ihrem Territorium nicht durch das FZA gebunden. Jedoch habe sie die völkervertraglich vereinbarten Bestimmungen des FZA zu beachten. ${ }^{22}$

Das Bundesgericht geht schliesslich kurz auf die Entstehungsgeschichte der Ausschaffungsinitiative ein, verweist auf eine
Auswahl von Voten im Parlament, und hält abschliessend fest, dass die Umsetzungsgesetzgebung der Ausschaffungsinitiative im Parlament ausführlich und kontrovers debattiert und kein Referendum dagegen ergriffen worden sei. ${ }^{23}$

26 Die Rüge des Beschwerdeführers, dass die Vorinstanz fälschlicherweise die Bestimmungen des StGB und nicht jene des FZA geprüft habe, taxiert es als nicht stichhaltig. Das FZA schreibe keine Prüfungsreihenfolge vor. Komme das Strafgericht landesrechtlich zu einem Ergebnis, das sich als mit dem FZA kompatibel erweise, sei das FZA offenkundig nicht verletzt. Die Frage der Normenhierarchie stelle sich gar nicht, wenn sich das Landesrecht völkerrechtskonform anwenden liesse. Die Frage der Normenhierarchie könne demnach offenbleiben. Das Strafgericht habe zunächst das ihm vertraute Landesrecht anzuwenden. ${ }^{24}$

27 Der Beschwerdeführer bringe weiter vor, dass gemäss Art. 5 Anhang I FZA unter Gesamtwürdigung der Umstände von der Anwesenheit des verurteilten Straftäters eine tatsächliche und hinreichend schwere Gefährdung der öffentlichen Sicherheit und Ordnung ausgehen müsse. Die dieser Rüge zugrundeliegende ausländerrechtliche Rechtsprechung sei jedoch nicht einschlägig, da sie vor dem Inkrafttreten von Art. 66a ff. StGB ergangen sei. Die Landesverweisung sei eine eigenständige strafrechtliche Massnahme. In casu sei keine Beendigung des Aufenthaltsrechts und keine Wegweisung im

\footnotetext{
23 Urteil des Bundesgerichts 6B_235/2018 vom 1. November 2018, E. 3.4.

24 Ebenda, E. 4.1.
}

Urteil des Bundesgerichts 6B_235/2018 vom 1. November 2018, E. 1.

22 Ebenda, E. 3.1. ff. mit Hinweis auf Art. 27 Wiener Übereinkommen vom 23. Mai 1969 über das Recht der Verträge (VRK; SR 0.111). 
Sinne des Ausländergesetzes ergangen, sondern eine Landesverweisung gemäss Art. $66 \mathrm{a}^{\text {bis }}$ StGB. ${ }^{25}$

Das Bundesgericht erwägt weiter, die von Volk und Ständen angenommene Initiative und deren Umsetzung führe mittels der strafrechtlichen Landesverweisung zu einer klaren Verschärfung der Praxis. So habe auch die federführende Bundespräsidentin in der Nationalratsdebatte von einer massiven Verschärfung der Ausschaffungspraxis gesprochen. ${ }^{26}$

29 Abschliessend hält es fest, dass, die Vorinstanz eine fallspezifische Prüfung der Rückfallgefahr vorgenommen und das Verhältnismässigkeitsprinzip angewendet habe. Die Beschwerde sei folglich abzuweisen.

\section{Kommentar}

30 Das Urteil des Bundesgerichts lässt den Leser perplex zurück. Erst nach mehrmaligem Lesen lassen sich die Kernaussagen herausschälen. Während die Schlussfolgerung, dass ein FZA-berechtigter verurteilter Straftäter nach Art. 66a ${ }^{\text {bis }}$ StGB im Einklang mit Art. 5 Anhang I FZA des Landes verwiesen werden $\mathrm{kann}^{27}$, grundsätzlich überzeugt, ist der Weg dahin von einigen irritierenden Erwägungen gepflastert. Dabei fallen insbesondere fünf Punkte auf:

25 Ebenda, E. 4.2.

26 Ebenda, E. 4.3.

27 Art. 66abis StGB lässt - im Gegensatz zum Automatismus der obligatorischen Landesverweisung nach Art. 66a StGB - durch seine Kann-Formulierung einen Auslegungsspielraum im Rahmen von Art. 5 Anhang I FZA zu. Es besteht hier folglich kein zwingender Normenkonflikt. Siehe hierzu Burri/Priuli (Fn. 5), S. 890.
31 Erstens befremdet die bundesgerichtliche Trennung von Rechtsbereichen, die der Gesetzgeber mit der Einführung der Art. 66a ff. StGB bewusst miteinander in Verbindung gesetzt hat. Die strafrechtliche Landesverweisung und deren ausländerrechtliche Wirkung sind unmittelbar miteinander verlinkt. So erlischt etwa gemäss Art. 61 Abs. 1 lit. e AIG28 die ausländerrechtliche Bewilligung mit einer nach Art. 66a StGB ausgesprochenen Landesverweisung. Gleichzeitig führen die obligatorische und die fakultative Landesverweisung zu einem Einreiseverbot (Art. 5 Abs. 1 lit. d AIG).29 Die Trennung der zwei Rechtsgebiete steht ferner im Widerspruch zu BGer 6B_209/2018 vom 23. November 2018, in welchem das Bundesgericht lediglich drei Wochen später auf die enge Verbindung zwischen der Landesverweisung und Bestimmungen im Ausländerrecht hinweist. 30

32 Zweitens ist kein sachlicher Grund ersichtlich, weshalb die im Ausländerrecht ergangene Rechtsprechung zu Art. 5 Anhang I FZA nicht weiterhin einschlägig sein soll. ${ }^{31}$ Tatsächlich ist diese vor Inkrafttreten von

$2 8 \longdiv { \text { Das Bundesgesetz über die Ausländerinnen und } }$ Ausländer (AuG) heisst seit dem 1. Januar 2019 «Bundesgesetz über die Ausländerinnen und Ausländer und über die Integration» (AIG; SR 142.20).

29 So auch Matthias Zurbrügg und Constantin Hruschka, in: Basler Kommentar, StGB und JStGB, 4. Auflage 2019, N. 58-60 und 86-93 zu Art. 66a-66d und Vetterli (Fn. 12).

30 Mit diesem Hinweis rechtfertigt das Bundesgericht in jenem Entscheid den Rückgriff auf das Migrationsrecht und die entsprechende Rechtsprechung im Kontext der Landesverweisung. BGer 6B_209/2018 vom 23. November 2018 (zur Publikation vorgesehen), E. 3.3.2.

31 Dabei irritiert besonders, dass das Bundesgericht im Urteil selbst mehrmals auf die im Ausländerrecht ergangene Rechtsprechung zu Art. 5 Anhang I FZA verweist (etwa E. 3.2. und E. 4.4.) und dadurch implizit die Rechtsprechung der öffentlich-rechtlichen Kammer, dass die Schweiz auf dem Gebiet des 
Art. 66a ff. StGB ergangen. Sie betrifft aber Art. 5 Anhang I FZA und nicht das StGB und dient daher in erster Linie der Auslegung von Art. 5 Anhang I FZA. Volk und Stände wie auch das Parlament haben durch die Annahme der Ausschaffungsinitiative und deren Umsetzung nicht die Auslegung von Art. 5 Anhang I FZA geändert. Die Voten und Resultate dieses Prozesses können zwar zur Auslegung von Art. 66a ff. StGB sowie zur Frage des Umgangs mit einem Normenkonflikt zwischen Landesverweisung und Völkerrecht beigezogen werden, nicht aber zur Auslegung von Art. 5 Anhang I FZA, die autonom erfolgt.

Drittens ist unbeachtlich, unter welchem gesetzlichen Titel die Schweiz Einschränkungen im Sinne von Art. 5 Anhang I FZA vornimmt. Art. 5 Anhang I FZA ist auf alle innerstaatlichen Einschränkungen des Aufenthaltsrechts von FZA-berechtigten Personen anwendbar, seien diese im StGB, im AIG oder in einem anderen Gesetz verankert. Wie vom Bundesgericht im Urteil richtig festgehalten, kann sich die Schweiz gemäss der Wiener Vertragsrechtskonvention nicht auf innerstaatliches Recht berufen, um sich nicht an die vereinbarten Bestimmungen des FZA halten zu müssen. Die fragliche Feststellung, dass das FZA keine strafrechtlichen Bestimmungen enthalte und deshalb kein strafrechtliches Abkommen sei, hat somit nicht zur Folge, dass das Bundesgericht oder dessen strafrechtliche Abteilung dieses nicht oder anders anzuwenden hat als bisher.

Ausländerrechts durch das FZA gebunden ist, anerkennt.
34 Viertens lässt das Urteil Begründungstiefe vermissen und enthält Widersprüche. So folgt etwa der Feststellung «kommt das Strafgericht landesrechtlich zu einem Ergebnis, das sich als mit dem FZA kompatibel erweist, ist das FZA offenkundig nicht verletzt» keine Erklärung. Namentlich erwägt das Bundesgericht nicht, weshalb das Ergebnis des Landesrechts (die vorliegende fakultative Landesverweisung) mit den Voraussetzungen von Art. 5 Anhang I FZA (nötige Gefährdung und Verhältnismässigkeit) kompatibel ist. Es lehnt die im Ausländerrecht ergangene Rechtsprechung zu Art. 5 Anhang I FZA zuerst ab, bietet aber keine anderen Auslegungshilfen der Bestimmung an und verweist im Folgenden wiederum auf die besagte Rechtsprechung. ${ }^{2}$ Gleichzeitig fehlt die Bundesgerichtsurteilen sonst immanente elegante Reduktion auf das Wesentliche. So enthält das Urteil kurze Verweise auf die parlamentarische Debatte und eine Aussage der «federführenden» Bundesrätin. Es ist jedoch nicht klar ersichtlich, welches sachliche Argument diese Einschübe untermauern sollen, denn eine Prüfung der Schubert-Praxis bleibt aus.

35 Und schliesslich haben Sätze wie «mit dem FZA vereinbarte die Schweiz - pointiert formuliert - keine Freizügigkeit für kriminelle Ausländer»; oder «das Strafgericht hat zunächst das ihm vertraute Landesrecht anzuwenden» einen ungewohnt politischen Unterton und wenig fachliche Überzeugungskraft.33 Bei wei-

32 E. 3.2. und E. 4.4. wiederum bestätigt in Urteil des Bundesgerichts 6B_209/2018 vom 23. November 2018 (zur Publikation vorgesehen).

33 Anderer Meinung Boris Etter, Bundesgericht, Strafrechtliche Abteilung, Urteil 6B_235/2018 vom 1. November 2018, X. gegen Oberstaatsanwaltschaft des Kantons Zürich, bedingter Straf- 
tem nicht jede Straftat rechtfertigt eine Einschränkung der Freizügigkeitsrechte. Und ein Gericht, im Besonderen das höchste Schweizer Gericht, hat das geltende, und nicht bloss das ihm vertraute Recht anzuwenden.

\section{Ausblick}

36 Was bleibt? Der Normenkonflikt zwischen der obligatorischen Landesverweisung und dem FZA ist nach wie vor nicht höchstrichterlich entschieden. Gemäss der bisherigen bundesgerichtlichen Rechtsprechung sowie der hier und andernorts vertretenen Meinung geht Art. 5 Anhang I FZA den Bestimmungen der neuen Landesverweisung vor.34 Die vorliegend besprochenen Urteile halten dem nichts entgegen, deuten jedoch an, dass der Konfliktbereich seitens des Bundesgerichts als eher gering eingeschätzt wird.

37 Rechtsanwendende Behörden haben folglich bei der Landesverweisung FZAberechtigter Ausländer nach wie vor Art. 5 Anhang I FZA zu prüfen. Dabei sollten sie sich auf die reiche bisherige Rechtsprechung der II. öffentlich-rechtlichen Kammer des Bundesgerichts zu Art. 5 Anhang I FZA stützen, welche viele Anhaltspunkte für

vollzug (qualifizierte einfache Körperverletzung), Landesverweisung (Art. 66a ${ }^{\text {bis }}$ StGB), AJP 2019 S. 244 ff., S. 246.

34 Vgl. BGE 142 II 35 E. 3.2 f.; BGE 133 V 367 E. 11.4 ff.; Zurbrügg/Hruschka (Fn. 29), N. 80 und $85 \mathrm{zu}$ Art. 66a-66d; Giusep Nay, Vorrang Völkerrecht: kein obiter dictum, kein Meinungsaustausch, in: Jusletter 18. April 2016; Astrid Epiney, Auslegung und Verhältnis des Freizügigkeitsabkommens zum nationalen Recht, in: Jusletter 14. März 2016. Dies wirkt sich entgegen der vom Bundesgericht in Urteil 6B_235/2018 vertretenen Meinung auf die Prüfungsreihenfolge einer zulässigen Landesverweisung aus. Siehe hierzu mit weiteren Hinweisen Burri/Priuli (Fn. 5); Gless/Petrig/Tobler (Fn. 5). die Gefährdungs- und Verhältnismässigkeitsprüfung geben kann. 35

38 Hierbei sollten sie ein spezielles Augenmerk auf die Prüfung legen, ob die betroffene Person überhaupt Ansprüche aus dem FZA ableiten kann. Fälschlicherweise wird in diesem Zusammenhang immer wieder auf «EU-Bürger» verwiesen - so auch von der II. Strafkammer des Zürcher Obergerichts. ${ }^{3}$ Die Gleichsetzung von «EU-Bürgern» und «freizügigkeitsberechtigten Personen» ist aber falsch, denn die Kreise der Freizügigkeitsberechtigten und der EU-Bürger sind in zweierlei Hinsicht nicht kongruent: Erstens können nicht alle EU-Bürger per se Ansprüche aus dem FZA ableiten, zweitens können auch Nicht-EU-Bürger (Drittstaatsangehörige) Ansprüche aus dem FZA ableiten. Es müssen daher in jedem Fall die spezifischen Voraussetzungen der im FZA aufgelisteten Einreise- oder Aufenthaltsrechte geprüft werden. 37

39 Letztendlich ist zu hoffen, dass das Bundesgericht in weiteren Urteilen, insbesondere betreffend die obligatorische Landesverweisung, überzeugendere Begründungen findet, die der Praxis aufzeigen, wie die Landesverweisung und das Personenfreizügigkeitsabkommen in der Schweiz rechtsdogmatisch miteinander in Einklang gebracht werden können. Falls es weiter daran festhalten will, dass Einschränkungen von Freizügigkeitsrechten gestützt auf das Strafgesetzbuch anders zu behandeln

35 Siehe etwa die Beispiele der Rechtsprechung nach gefährdetem Rechtsgut geordnet in Burri/Priuli (Fn. 5), S. $893 \mathrm{ff}$.

36 Urteil des Zürcher Obergerichts (II. Strafkammer) SB170315 vom 16. Januar 2018 E. IV.2.c). Ferner auch wiederholt Etter (Fn. 33), S. 248-250.

37 Wie eingangs erwähnt gilt Dasselbe für das EFTA-Abkommen. Siehe Gless/Petrig/Tobler (Fn. 5), S. 101 f.; Burri/Priuli (Fn. 5), S. 888. 
sind als solche, die gestützt auf das Ausländerrecht verfügt werden, so wird es dies noch begründen müssen. 